Contributions to Game Theory and Management, XIV, 8-19

\title{
Static and Dynamic Game Theoretic Models of Opinion Control in Marketing Networks*
}

\author{
Movlatkhan T. Agieva ${ }^{1}$, Alexey V. Korolev ${ }^{2}$ and \\ Guennady A. Ougolnitsky ${ }^{3}$ \\ 1 Ingush State University, \\ Magistralnaya St. 39, Nazran, 386132, Russia \\ E-mail: agieva25@mail.ru \\ 2 National Research University \\ Higher School of Economics at St. Petersburg, \\ Kantemirovskaya St. 3A, St. Petersburg, 190008, Russia \\ E-mail: danitschi@gmail.com \\ 3 Southern Federal University, \\ J.I. Vorovich Institute of Mathematics, Mechanics and Computer Sciences, \\ Milchakov St. 8a, Rostov-on-Don, 344090, Russia \\ E-mail: gaugolnickiy@sfedu.ru
}

\begin{abstract}
In this paper we consider game theoretic models of control on networks with application to marketing. We suppose that all strong subgroups are determined in the stage of analysis of the influence digraph, and the control impact is exerted only to the members of those subgroups because they determine all stable final opinions. An agent's opinion is interpreted as his expenses for buying goods (services) of a firm. The following problem of opinion control is being studied. A dynamic (difference) game in normal form where the players solve the problem of maximization of the sum of opinions of the members of a target audience by means of the closed-loop strategies of impact to the current opinions of the members of strong subgroups. We received the analytical solutions and conducted their comparative analysis.

Keywords: games in normal form, models of impact and control on networks, marketing.
\end{abstract}

\section{Introduction}

A basic model of influence in a social group was first considered in (French, 1956), (Harary, 1959) and studied in more details in (De Groot, 1974). Then many generalizations and refinements of this model were considered: dynamic interactions, conditions of convergence, speed of convergence, conditions of uniqueness of the final opinion, and so on (De Marzo et al., 2003), (Golub and Jackson, 2010), (Hegselman and Krause, 2002). One of the most detailed monographs on network modeling is (Jackson, 2008); see also (Jackson and Wolinsky, 1996). An application of the network models to the analysis of political processes is described in the monograph (Aleskerov et al., 2007). The issues of communication and coordination in social networks are analyzed in (Chwe, 2000). The (Godes D., Mayzlin, 2004), (Goldenberg et al., 2001) are concerned with the "word of mouth". A detailed analysis of the models of influence in networks is presented in (Gubanov, 2011), (Chkhartishvili et al., 2019).

Together with models of influence, the models of control in social groups with given structure of interactions are of even more interests (Chkhartishvili et al., 2019).

${ }^{*}$ The work was supported by the Russian Science Foundation, project № 17-19-01038.

https://doi.org/10.21638/11701/spbu31.2021.01 
In turn, it is natural here to differentiate the models of optimal control (the only control agent) and the models of conflict control (several interacting control agents with different interests). A mathematical formalization of the latter models generates game theoretic setups on networks (Novikov, 2014), (Jackson and Zenou, 2014). The differential game theoretic models in marketing are presented in the monograph (Jorgensen and Zaccour, 2004) and review (Jorgensen and Zaccour, 2014).

In this paper we investigate an opinion control problem with application to marketing. This is a dynamic (difference) game in normal form, where the players maximize the sum of opinions of the members of a target audience by the closed-loop strategies of impact to the current opinions of the members of strong subgroups. The analytical solutions are received, and their comparative analysis is made.

Lagrange multipliers method is used to find players' strategies. This method is used at each step of the iterative procedure for a control impact of the $i$-th firm to the $j$-th agent in the $t$-th period of time.

\section{The principal information}

The basic model of influence in a social network is a weighted directed graph in which the vertices correspond to the members of the social group (agents), and the arcs describe their mutual interaction. Each vertex is ascribed a real value (an opinion of the member of the group) that can change in time, and each arc is ascribed another real value (a weight) that characterizes a degree of influence of one agent to another (or, that is the same, a degree of trust of the latter agent to the former one).

Thus, a network is modeled by a digraph $D=(Y, A)$, where $Y=\left\{y_{1}, \ldots, y_{n}\right\}$ is a set of agents (vertices); $y_{i} \rightarrow x_{i}(t), i=1, \ldots, n$ - the agent's opinion; $x^{0}=$ $\left(x_{1}^{0}, \ldots, x_{n}^{0}\right)$ - a vector of initial opinions of the agents; $a_{i j}$ - a coefficient of influence of the $i$-th agent to the opinion of the $j$-th agent; $A=\left\|a_{i j}\right\|$ - a matrix of influences (determines the set of arcs of the network model). The opinions dynamics is defined by the rule

$$
x_{j}^{t+1}=\sum_{i=1}^{n} a_{i j} x_{i}^{t}, x_{j}^{0}=x_{j 0}, \quad j=1, \ldots, n .
$$

In (Roberts, 1976) it is shown that all members of each i-th strong subgroup (a nondegenerated strong component of the network that belongs to the vertex base of its condensation) come with time to the common stable final opinion defined by the formula

$$
x_{i}^{\infty}=\sum_{k=1}^{n_{i}} w_{k}^{(i)} x_{k 0}^{(i)}
$$

where $w_{k}^{(i)}-$ a component of the stationary vector for the Markov chain with the transition matrix $A_{i}^{T}, n_{i}$ - a number of members of the $i$-th strong subgroup. The final opinions of other agents that do not belong to any strong subgroup ("companions") are calculated as

$$
x_{j}^{\infty}=\sum_{i=1}^{r} b_{j i} x_{i}^{\infty},
$$

where $b_{j i}$ - a probability of transition of the agent $j$ to the strong subgroup $i$ as an ergodic set of states of the Markov chain, $r$ - a total number of strong subgroups in the digraph of influences. 
Consider an application of the models of influence and control on net-works to marketing. Our approach is presented in (Agieva et al., 2019), (Korolev and Ougolnitsky, 2020), (Agieva et al., 2020). There are $m$ firms that are capable to exert influence to a target audience for the change of its opinion to the desirable direction. At least one of the firms realizes marketing actions that are considered as an impact to the opinions of the target audience. The pay-off of each firm consists in the increase of the opinions. An agent's opinion is treated as the total costs of buying goods (services) of a firm - one of the agents of conflict control.

Let us study different game theoretic control problems setups. It is assumed that the strong subgroups are already found, and only the members of strong subgroups are influenced. Let us illustrate the solutions on the following test example (Fig.1).

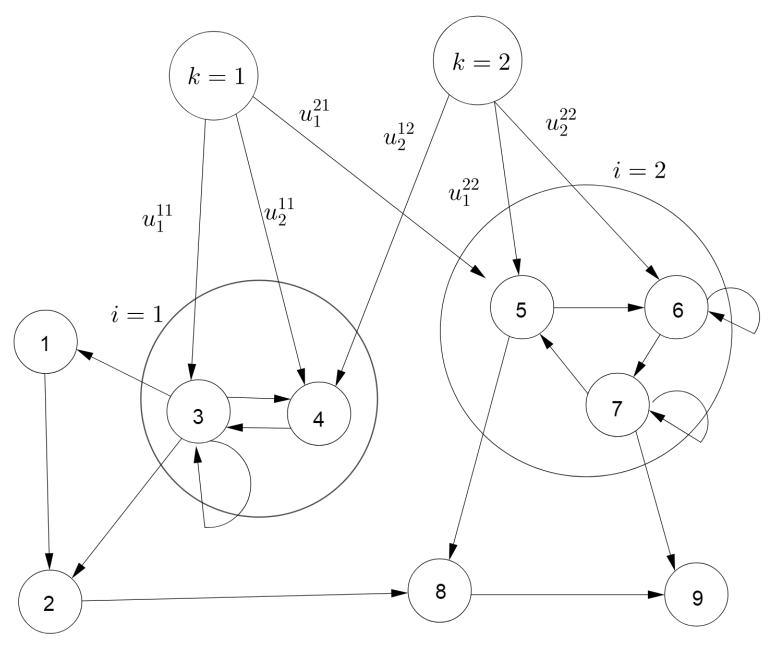

Fig. 1. A test example. The market consists of 9 buyers (agents). Their effects on each other are shown by arrows. There are two strong subgroups $(i=1,2)$. There are two firms $(k=1,2)$ that exert a control influence on the opinions of buyers. The control effect of the $k$-th firm on the $j$-th member of the $i$-th strong subgroup is denoted by $u_{j}^{i, k}$

\section{Dynamic model}

The of impact in this model is to maximize the sum of opinions of the members of target audience for the whole period from $t=1$ till $t=n$. The impact in closed-loop strategies is exerted on the current opinions of the members of strong subgroups.

\subsection{Independent behavior of the players}

There are $N$ agents and $m$ firms. Each $i$-th firm solves the following optimization problem:

$$
\sum_{t=1}^{n}\left[e^{-\rho(t-1)} \sum_{j=1}^{N}\left(x_{j}^{t}+\sum_{i=1}^{m} u_{j}^{i(t)}\left(x_{j}^{t}\right)\right)\right] \rightarrow \max
$$




$$
\begin{gathered}
x_{j}^{t+1}=\sum_{l=1}^{N} a_{l j}\left(x_{l}^{t}+\sum_{i=1}^{m} u_{l}^{i(t)}\left(x_{l}^{t}\right)\right), x_{j}^{0}=x_{j 0}, j=1,2, \ldots, N, t=1,2, \ldots, n-1, \\
\sum_{t=1}^{n} e^{-\rho(t-1)} \sum_{j=1}^{N}\left[u_{j}^{i(t)}\left(x_{j}^{t}\right)\right]^{p}=R_{i}, \\
u_{j}^{i(t)}\left(x_{j}^{t}\right) \geq 0, j=1,2, \ldots, N, t=1,2, \ldots, n,
\end{gathered}
$$

where $u_{j}^{i(t)}\left(x_{j}^{t}\right)$ - a control impact of the $i$-th firm to the $j$-th agent in the $t$-th period of time. This value is positive if the $j$-th agent enters into one of the strong subgroups and is one of the agents in this subgroup who are impacted by the $i$-th firm, otherwise $u_{j}^{i(t)}\left(x_{j}^{t}\right)=0$. The total (by all strong subgroups of the network) number of agents impacted by the $i$-th firm is denoted by $m_{i}$.

It is more convenient to describe the problem setup and its solution in a matrix form. The problem for the $i$-th firm is

$$
\begin{gathered}
\varepsilon \sum_{t=1}^{n} \delta^{t-1}\left(X^{t}+B u^{i(t)}\right) \rightarrow \max \\
X^{t+1}=A^{T}\left[X^{t}+B u^{i(t)}\right], t=1,2, \ldots, n-1, X^{1}=X, \\
u^{i(t)} \geq 0, t=1,2, \ldots, n, \\
\sum_{t=1}^{n} \delta^{t-1} \sum_{j=1}^{N}\left(u_{j}^{i(t)}\left(x_{j}^{t}\right)\right)^{p}=R_{i},
\end{gathered}
$$

where $T$ stands for transposing, $\sum_{j=1}^{N}\left(u_{j}^{i(t)}\left(x_{j}^{t}\right)\right)^{p}$ - a sum of the control impacts of the $i$-th firm in the $t$-th period. The matrix $B$ consists of $m$ blocks that is number of firms. Each block $B_{i}$ describes impacts of the $i$-th firm and has dimension $N \times m_{i}$, i.e. its number of rows is equal to the number of agents in the network, and the number of columns is equal to the total number of members of the strong subgroups that are impacted. The respective element of the matrix $B$ is equal to one if the impact holds, and to zero, otherwise. $X-$ a column vector of the values of state variables, $u$ - a column vector of the control impacts of the firms, $\varepsilon$ - a row vector from units of the length $N, \delta$ denotes a discount factor, i.e. $\delta=e^{-\rho}$.

Consider a one-step game (the recursion base).The $i$-th firm maximizes the function

$$
\varepsilon\{X+B u\} \rightarrow \max
$$

with constraint

$$
\sum_{j=1}^{N}\left(u_{j}^{i}\left(x_{j}\right)\right)^{p}=R_{i} .
$$


Solving this optimization problem we receive the relations

$$
\frac{1}{1}=\left(\frac{u_{j_{1}}^{i}}{u_{j_{2}}^{i}}\right)^{p-1}
$$

for any agents $j_{1}$ and $j_{2}$ that are impacted by the $i$-th firm. Substituting (5) in (4), we receive for all agents $j$ impacted by the firm i:

$$
u_{j}^{i}=\sqrt[p]{\frac{R_{i}}{m_{i}}} .
$$

Consider a two-step game. Each $i$-th firm solves the problem

$$
\varepsilon\left\{X+B u+\delta\left[A^{T}(X+B u)+B u^{*}\right]\right\} \rightarrow \max
$$

where $u^{*}$ - the solution of the one-step game, with constraint

$$
\sum_{j=1}^{N}\left(u_{j}^{i}\right)^{p}=R_{i}
$$

Solving this optimization problem we receive the relations

$$
\frac{1+\delta A_{j_{1}}}{1+\delta A_{j_{2}}}=\left(\frac{u_{j_{1}}^{i}}{u_{j_{2}}^{i}}\right)^{p-1}
$$

for any agents $j_{1}$ and $j_{2}$ that are impacted by the $i$-th firm, where $A_{j}$ denotes the sum of elements of the $j$-th row of the influence matrix $A$. Substitution of (7) to (6) gives the solution

$$
u_{j}^{i}=\sqrt[p]{\frac{R_{i}}{\sum_{k=1}^{m_{i}}\left(1+\delta A_{k}\right)^{\frac{p}{p-1}}}}\left(1+\delta A_{j}\right)^{\frac{1}{p-1}} .
$$

Consider a three-step game. Each $i$-th firm solves the problem

$\varepsilon\left\{X+B u+\delta\left[A^{T}(X+B u)+B u^{* *}\right]+\delta^{2}\left[A^{T}\left(A^{T}(X+B u)+B u^{* *}\right)+B u^{*}\right]\right\} \rightarrow \max$

with constraint

$$
\sum_{j=1}^{N}\left(u_{j}^{i}\right)^{p}=R_{i}
$$

where $u^{*}$ - the solution of the one-step game, $u^{* *}$ - the solution of the two-step game. The same actions as in the two-step case give the relations

$$
\frac{1+\delta A_{j_{1}}+\delta^{2} A_{j_{1}}^{2}}{1+\delta A_{j_{2}}+\delta^{2} A_{j_{2}}^{2}}=\left(\frac{u_{j_{1}}^{i}}{u_{j_{2}}^{i}}\right)^{p-1}
$$

for any agents $j_{1}$ and $j_{2}$ that are impacted by the $i$-th firm, where $A_{j}^{2}$ denotes the sum of elements of the $j$-th row of the square of the influence matrix. In general, 
denote by $A_{j}^{s}$ the sum of elements of the $j$-th row of the $s$-th power of the matrix $A$. The substitution of the received relations (9) into the budget con-straint (8) gives the solution

$$
u_{j}^{i}=\sqrt[p]{\frac{R_{i}}{\sum_{k=1}^{m_{i}}\left(1+\delta A_{k}+\delta^{2} A_{k}^{2}\right)^{\frac{p}{p-1}}}}\left(1+\delta A_{j}+\delta^{2} A_{j}^{2}\right)^{\frac{1}{p-1}} .
$$

Repeating this process, we receive for the $n$-step game

$$
\begin{aligned}
& u_{j}^{i}= \\
& \sqrt[p]{\frac{R_{i}}{\sum_{k=1}^{m_{i}}\left(1+\delta A_{k}+\delta^{2} A_{k}^{2}+\cdots+\delta^{n-1} A_{k}^{n-1}\right)^{\frac{p}{p-1}}}}\left(1+\delta A_{j}+\delta^{2} A_{j}^{2}+\cdots+\delta^{n-1} A_{j}^{n-1}\right)^{\frac{1}{p-1}} .
\end{aligned}
$$

The summing by $k$ in the denominator is made by all agents of strong subgroups that are impacted by the $i$-th firm.

Let us write down the payoff of each firm. If any firm does not exert impact to any agent, the payoff is equal to

$$
\varepsilon\left[I+\delta A^{T}+\delta^{2}\left(A^{T}\right)^{2}+\ldots+\delta^{n-1}\left(A^{T}\right)^{n-1}\right] X=\varepsilon\left[I-\left(\delta A^{T}\right)^{n}\right]\left(I-\delta A^{T}\right)^{-1} X .
$$

If at least one firm impacts to a set of agents from the strong subgroups then the payoff of each firm increases. Let us calculate it sequentially. If in the first period the $i$-th firm solves the $n$-step problem then the payoff of each firm increases by the value

$$
\begin{aligned}
& \sum_{j=1}^{m_{i}} \sum_{s=0}^{n-1} \delta^{s} A_{j}^{s} \sqrt[p]{\frac{R_{i}^{(1)}}{\sum_{k=1}^{m_{i}}\left(\sum_{s=0}^{n-1} \delta^{s} A_{k}^{s}\right)^{\frac{p}{p-1}}}}\left(\sum_{s=1}^{n-1} \delta^{s} A_{j}^{s}\right)^{\frac{1}{p-1}}= \\
& =\sum_{j=1}^{m_{i}} \sqrt[p]{\frac{R_{i}^{(1)}}{\sum_{k=1}^{m_{i}}\left(\sum_{s=0}^{n-1} \delta^{s} A_{k}^{s}\right)^{\frac{p}{p-1}}}}\left(\sum_{s=1}^{n-1} \delta^{s} A_{j}^{s}\right)^{\frac{p}{p-1}}= \\
& =\sqrt[p]{\frac{R_{i}^{(1)}}{\sum_{k=1}^{m_{i}}\left(\sum_{s=0}^{n-1} \delta^{s} A_{k}^{s}\right)^{\frac{p}{p-1}}} \sum_{j=1}^{m_{i}}\left(\sum_{s=1}^{n-1} \delta^{s} A_{j}^{s}\right)^{\frac{p}{p-1}}}= \\
& =\sqrt[p]{R_{i}^{(1)}}\left[\sum_{j=1}^{m_{i}}\left(\sum_{s=0}^{n-1} \delta^{s} A_{j}^{s}\right)^{\frac{p}{p-1}}\right]^{\frac{p-1}{p}},
\end{aligned}
$$

where $R_{i}^{(1)}$ - a budget of the $i$-th firm in the first period of time. Here and elsewhere the denotation $\sum_{j=1}^{m_{i}}$ or $\sum_{k=1}^{m_{i}}$ means that the summing is made by all agents 
impacted by the $i$-th firm. Similarly, in the second period the $i$-th firm solves $(n-1)$ step optimization problem, and all firms receive the additional payoff

$$
\sqrt[p]{R_{i}^{(2)}}\left[\sum_{j=1}^{m_{i}}\left(\sum_{s=0}^{n-2} \delta^{s} A_{j}^{s}\right)^{\frac{p}{p-1}}\right]^{\frac{p-1}{p}} \delta,
$$

and so on. In the $q$-th period of time the $i$-th firm solves $(n-q+1)$-step optimization problem, and the payoff of each firm is augmented by the value

$$
\sqrt[p]{R_{i}^{(q)}}\left[\sum_{j=1}^{m_{i}}\left(\sum_{s=0}^{n-q} \delta^{s} A_{j}^{s}\right)^{\frac{p}{p-1}}\right]^{\frac{p-1}{p}} \delta^{q-1} .
$$

In the $(n-2)$-th period of time the $i$-th firm solves the three-step optimization problem, and the payoff of each firm is augmented by the value

$$
\sqrt[p]{R_{i}^{(n-2)}}\left[\sum_{j=1}^{m_{i}}\left(1+\delta A_{j}+\delta^{2} A_{j}^{2}\right)^{\frac{p}{p-1}}\right]^{\frac{p-1}{p}} \delta^{n-3} .
$$

In the $(n-1)$-th period of time the $i$-th firm solves the two-step optimization problem, and the payoff of each firm is augmented by the value

$$
\sqrt[p]{R_{i}^{(n-1)}}\left[\sum_{j=1}^{m_{i}}\left(1+\delta A_{j}\right)^{\frac{p}{p-1}}\right]^{\frac{p-1}{p}} \delta^{n-2} .
$$

In the $n$-th period of time the $i$-th firm solves the one-step optimization problem, and the payoff of each firm is augmented by the value

$$
\sqrt[p]{R_{i}^{(n)}}\left(m_{i}\right)^{\frac{p-1}{p}} \delta^{n-1} .
$$

Now it stays to allocate the budget between the time periods from the 1-st till the $n$-th, i.e. to maximize the expression

$$
\begin{gathered}
\sqrt[p]{R_{i}^{(1)}\left[\sum_{j=1}^{m_{i}}\left(\sum_{s=0}^{n-1} \delta^{s} A_{j}^{s}\right)^{\frac{p}{p-1}}\right]^{\frac{p-1}{p}}}+\ldots+\sqrt[p]{R_{i}^{(n-1)}} \delta^{n-2}\left[\sum_{j=1}^{m_{i}}\left(\sum_{s=0}^{1} \delta^{s} A_{j}^{s}\right)^{\frac{p}{p-1}}\right]^{\frac{p-1}{p}}+ \\
+\sqrt[p]{R_{i}^{(n)}\left(m_{i}\right)^{p-1}} \delta^{n-1}
\end{gathered}
$$

with constraint

$$
R_{i}^{(1)}+\delta R_{i}^{(2)}+\ldots+\delta^{n-2} R_{i}^{(n-1)}+\delta^{n-1} R_{i}^{(n)}=R_{i} .
$$

The solution of this problem gives that for any $q=1,2, \ldots, n$ the equality holds

$$
\frac{\left[\sum_{j=1}^{m_{i}}\left(\sum_{s=0}^{n-q} \delta^{s} A_{j}^{s}\right)^{\frac{p}{p-1}}\right]^{\frac{p-1}{p}} \delta^{q-1}}{p\left[R_{i}^{(q)}\right]^{\frac{p-1}{p}}}=\lambda \delta^{q-1},
$$


that implies that the budget should be allocated in time according the ratio

$$
\frac{R_{i}^{\left(q_{1}\right)}}{R_{i}^{\left(q_{2}\right)}}=\frac{\sum_{j=1}^{m_{i}}\left(\sum_{s=0}^{n-q_{1}} \delta^{s} A_{j}^{s}\right)^{\frac{p}{p-1}}}{\sum_{j=1}^{m_{i}}\left(\sum_{s=0}^{n-q_{2}} \delta^{s} A_{j}^{s}\right)^{\frac{p}{p-1}}}
$$

where $1 \leq q_{1}, q_{2} \leq n$. Expressing the budgets of all periods by the first-period budget and substituting in the common budget constraint (11), we receive

$$
\begin{gathered}
R_{i}^{(1)}\left(\frac{\sum_{j=1}^{m_{i}}\left(\sum_{s=0}^{n-1} \delta^{s} A_{j}^{s}\right)^{\frac{p}{p-1}}}{m_{i}}+\ldots+\delta^{n-3} \frac{\sum_{j=1}^{m_{i}}\left(\sum_{s=0}^{2} \delta^{s} A_{j}^{s}\right)^{\frac{p}{p-1}}}{m_{i}}+\right. \\
\left.+\delta^{n-2} \frac{\sum_{j=1}^{m_{i}}\left(\sum_{s=0}^{1} \delta^{s} A_{j}^{s}\right)^{\frac{p}{p-1}}}{m_{i}}+\delta^{n-1}\right)=R_{i},
\end{gathered}
$$

that gives the optimal allocation of the budget of the $i$-th firm in time periods

$$
R_{i}^{(q)}=\frac{\sum_{j=1}^{m_{i}}\left(\sum_{s=0}^{n-q} \delta^{s} A_{j}^{s}\right)^{\frac{p}{p-1}}}{\sum_{j=1}^{m_{i}}\left(\sum_{s=0}^{n-1} \delta^{s} A_{j}^{s}\right)^{\frac{p}{p-1}}+\ldots+\delta^{n-2} \sum_{j=1}^{m_{i}}\left(\sum_{s=0}^{1} \delta^{s} A_{j}^{s}\right)^{\frac{p}{p-1}}+\delta^{n-1} m_{i}} R_{i} .
$$

The substitution of the expression (12) in the formula (10) gives a final payoff of each firm when in the $q$-th $(q=1,2, \ldots, n)$ period the $i$-th firm solves $(n-q+1)$-step optimization problem

$$
\begin{gathered}
\frac{\sqrt[p]{R_{i}}\left[\sum_{j=1}^{m_{i}}\left(\sum_{s=0}^{n-q} \delta^{s} A_{j}^{s}\right)^{\frac{p}{p-1}}\right]^{\frac{1}{p}}\left[\sum_{j=1}^{m_{i}}\left(\sum_{s=0}^{n-q} \delta^{s} A_{j}^{s}\right)^{\frac{p}{p-1}}\right]^{\frac{p-1}{p}} \delta^{q-1}}{\sqrt[p]{\sum_{j=1}^{m_{i}}\left(\sum_{s=0}^{n-1} \delta^{s} A_{j}^{s}\right)^{\frac{p}{p-1}}+\ldots+\delta^{n-2} \sum_{j=1}^{m_{i}}\left(\sum_{s=0}^{1} \delta^{s} A_{j}^{s}\right)^{\frac{p}{p-1}}+\delta^{n-1} m_{i}}}= \\
=\frac{\sqrt[p]{R_{i}} \sum_{j=1}^{m_{i}}\left(\sum_{s=0}^{n-q} \delta^{s} A_{j}^{s}\right)^{\frac{p}{p-1}} \delta^{q-1}}{\sqrt[p]{\sum_{j=1}^{m_{i}}\left(\sum_{s=0}^{n-1} \delta^{s} A_{j}^{s}\right)^{\frac{p}{p-1}}+\ldots+\delta^{n-2} \sum_{j=1}^{m_{i}}\left(\sum_{s=0}^{1} \delta^{s} A_{j}^{s}\right)^{\frac{p}{p-1}}+\delta^{n-1} m_{i}}} .
\end{gathered}
$$

Summing the payoffs of each firm from the efforts of the $i$-th firm in control of the target audience in all $\mathrm{n}$ periods of time, we receive

$$
\frac{\sqrt[p]{R_{i}}\left\{\sum_{j=1}^{m_{i}}\left(\sum_{s=0}^{n-1} \delta^{s} A_{j}^{s}\right)^{\frac{p}{p-1}}+\ldots+\delta^{n-2} \sum_{j=1}^{m_{i}}\left(\sum_{s=0}^{1} \delta^{s} A_{j}^{s}\right)^{\frac{p}{p-1}}+\delta^{n-1} m_{i}\right\}}{\sqrt[p]{\sum_{j=1}^{m_{i}}\left(\sum_{s=0}^{n-1} \delta^{s} A_{j}^{s}\right)^{\frac{p}{p-1}}+\ldots+\delta^{n-2} \sum_{j=1}^{m_{i}}\left(\sum_{s=0}^{1} \delta^{s} A_{j}^{s}\right)^{\frac{p}{p-1}}+\delta^{n-1} m_{i}}}=
$$




$$
=\sqrt[p]{R_{i}}\left[\sum_{j=1}^{m_{i}}\left(\sum_{s=0}^{n-1} \delta^{s} A_{j}^{s}\right)^{\frac{p}{p-1}}+\ldots+\delta^{n-2} \sum_{j=1}^{m_{i}}\left(\sum_{s=0}^{1} \delta^{s} A_{j}^{s}\right)^{\frac{p}{p-1}}+\delta^{n-1} m_{i}\right]^{\frac{p-1}{p}} .
$$

Summing the control efforts of all firms, we receive the payoff of each $i$-th firm

$$
\begin{gathered}
\varepsilon\left[I+\delta A^{T}+\delta^{2}\left(A^{T}\right)^{2}+\ldots+\delta^{n-1}\left(A^{T}\right)^{n-1}\right] X-R_{i}+ \\
+\sum_{i=1}^{m}\left\{\sqrt[p]{R_{i}}\left[\sum_{j=1}^{m_{i}}\left(\sum_{s=0}^{n-1} \delta^{s} A_{j}^{s}\right)^{\frac{p}{p-1}}+\ldots+\delta^{n-2} \sum_{j=1}^{m_{i}}\left(\sum_{s=0}^{1} \delta^{s} A_{j}^{s}\right)^{\frac{p}{p-1}}+\delta^{n-1} m_{i}\right]^{\frac{p-1}{p}}\right\} .
\end{gathered}
$$

Respectively, the total payoff of all firms is equal to

$$
\begin{gathered}
\varepsilon\left[I+\delta A^{T}+\delta^{2}\left(A^{T}\right)^{2}+\ldots+\delta^{n-1}\left(A^{T}\right)^{n-1}\right] X-\sum_{i=1}^{m} R_{i}+ \\
+\sum_{i=1}^{m}\left\{\sqrt[p]{R_{i}}\left[\sum_{j=1}^{m_{i}}\left(\sum_{s=0}^{n-1} \delta^{s} A_{j}^{s}\right)^{\frac{p}{p-1}}+\ldots+\delta^{n-2} \sum_{j=1}^{m_{i}}\left(\sum_{s=0}^{1} \delta^{s} A_{j}^{s}\right)^{\frac{p}{p-1}}+\delta^{n-1} m_{i}\right]^{\frac{p-1}{p}}\right\} .
\end{gathered}
$$

\subsection{Cooperative behavior of the players}

There are $N$ agents and $m$ firms. A control body in the name of all firms solves the following optimization problem:

$$
\begin{gathered}
\sum_{t=1}^{n}\left[e^{-\rho(t-1)} \sum_{j=1}^{N}\left(x_{j}^{t}+\sum_{i=1}^{m} u_{j}^{i(t)}\left(x_{j}^{t}\right)\right)\right] \rightarrow \max , \\
x_{j}^{t+1}=\sum_{l=1}^{N} a_{l j}\left(x_{l}^{t}+\sum_{i=1}^{m} u_{l}^{i(t)}\left(x_{l}^{t}\right)\right), x_{j}^{0}=x_{j 0}, j=1,2, \ldots, N, t=1,2, \ldots, n-1, \\
\sum_{t=1}^{n}\left\{e^{-\rho(t-1)} \sum_{i=1}^{m} \sum_{j=1}^{N}\left[u_{j}^{i(t)}\left(x_{j}^{t}\right)\right]^{p}\right\}=R, R=\sum_{i=1}^{m} R_{i}, \\
u_{j}^{i(t)}\left(x_{j}^{t}\right) \geq 0, j=1,2, \ldots, N, i=1,2, \ldots, m, t=1,2, \ldots, n,
\end{gathered}
$$

where $u_{j}^{i(t)}\left(x_{j}^{t}\right)$ is the value of impact of the $i$-th firm on the $j$-th agent in the $t$-th period of time. This value is positive if the $j$-th agent enters into one of the strong subgroups and is one of the agents in this subgroup who are impacted by the $i$-th firm, otherwise $u_{j}^{i(t)}\left(x_{j}^{t}\right)=0$. Denote $m_{i}$ total number of agents im-pacted by the $i$-th firm.

The cooperative solution is technically the same as in the case of independent players, so we give at once the received evident result.

The total payoff is equal to

$$
\varepsilon\left[I+\delta A^{T}+\delta^{2}\left(A^{T}\right)^{2}+\ldots+\delta^{n-1}\left(A^{T}\right)^{n-1}\right] X-\sum_{i=1}^{m} R_{i}+
$$


$+\sqrt[p]{\sum_{i=1}^{m} R_{i}}\left\{\sum_{i=1}^{m}\left[\sum_{j=1}^{m_{i}}\left(\sum_{s=0}^{n-1} \delta^{s} A_{j}^{s}\right)^{\frac{p}{p-1}}+\ldots+\delta^{n-2} \sum_{j=1}^{m_{i}}\left(\sum_{s=0}^{1} \delta^{s} A_{j}^{s}\right)^{\frac{p}{p-1}}+\delta^{n-1} m_{i}\right]\right\}^{\frac{p-1}{p}}$.

The difference between the total payoffs in the cooperative and independent cases is equal to

$$
\begin{aligned}
& \sqrt[p]{\sum_{i=1}^{m} R_{i}}\left\{\sum_{i=1}^{m}\left[\sum_{j=1}^{m_{i}}\left(\sum_{s=0}^{n-1} \delta^{s} A_{j}^{s}\right)^{\frac{p}{p-1}}+\ldots+\delta^{n-2} \sum_{j=1}^{m_{i}}\left(\sum_{s=0}^{1} \delta^{s} A_{j}^{s}\right)^{\frac{p}{p-1}}+\delta^{n-1} m_{i}\right]\right\}^{\frac{p-1}{p}}- \\
& -\sum_{i=1}^{m}\left\{\sqrt[p]{R_{i}}\left[\sum_{j=1}^{m_{i}}\left(\sum_{s=0}^{n-1} \delta^{s} A_{j}^{s}\right)^{\frac{p}{p-1}}+\ldots+\delta^{n-2} \sum_{j=1}^{m_{i}}\left(\sum_{s=0}^{1} \delta^{s} A_{j}^{s}\right)^{\frac{p}{p-1}}+\delta^{n-1} m_{i}\right]^{\frac{p-1}{p}}\right\} .
\end{aligned}
$$

Lemma 1. When $p>1$ the value (14) is non-negative, and when $p<1$ it is non-positive.

Proof. Denote:

$$
q=\frac{p}{p-1}, S_{i}=\sum_{j=1}^{m_{i}}\left(\sum_{s=0}^{n-1} \delta^{s} A_{j}^{s}\right)^{\frac{p}{p-1}}+\ldots+\delta^{n-2} \sum_{j=1}^{m_{i}}\left(\sum_{s=0}^{1} \delta^{s} A_{j}^{s}\right)^{\frac{p}{p-1}}+\delta^{n-1} m_{i} .
$$

Then the expression (14) takes the form

$$
\left(\sum_{i=1}^{m} R_{i}\right)^{\frac{1}{p}}\left(\sum_{i=1}^{m} S_{i}\right)^{\frac{1}{q}}-\sum_{i=1}^{m}\left(R_{i}\right)^{\frac{1}{p}}\left(S_{i}\right)^{\frac{1}{q}} .
$$

According the Holder inequality, if $p>1$ and $\frac{1}{p}+\frac{1}{q}=1$ then

$$
\sum_{i=1}^{m} a_{i} b_{i} \leq\left(\sum_{i=1}^{m} a_{i}^{p}\right)^{\frac{1}{p}}\left(\sum_{i=1}^{m} b_{i}^{q}\right)^{\frac{1}{q}}
$$

or, that is the same,

$$
\sum_{i=1}^{m}\left(a_{i}\right)^{\frac{1}{p}}\left(b_{i}\right)^{\frac{1}{q}} \leq\left(\sum_{i=1}^{m} a_{i}\right)^{\frac{1}{p}}\left(\sum_{i=1}^{m} b_{i}\right)^{\frac{1}{q}} .
$$

Taking in the Holder inequality $a_{i}=R_{i}, b_{i}=S_{i}$, we receive when $p>1$ :

$$
\left(\sum_{i=1}^{m} R_{i}\right)^{\frac{1}{p}}\left(\sum_{i=1}^{m} S_{i}\right)^{\frac{1}{q}}-\sum_{i=1}^{m}\left(R_{i}\right)^{\frac{1}{p}}\left(S_{i}\right)^{\frac{1}{q}} \geq 0 .
$$

Accordingly, in the case of $p<1$ from the Holder inequality, we have

$$
\left(\sum_{i=1}^{m} R_{i}\right)^{\frac{1}{p}}\left(\sum_{i=1}^{m} S_{i}\right)^{\frac{1}{q}}-\sum_{i=1}^{m}\left(R_{i}\right)^{\frac{1}{p}}\left(S_{i}\right)^{\frac{1}{q}} \leq 0 .
$$

Thus, from the point of view of the total payoff the cooperation is more advantageous than competition when the control cost is big, and vice versa, when the control cost is small. 


\section{Conclusion}

In this paper we consider game theoretic models of control on networks with application to marketing. We suppose that all strong subgroups are determined in the stage of analysis of the influence digraph, and the control impact is exerted only to the members of those subgroups because they determine all stable final opinions. An agent's opinion is interpreted as his expenses for buying goods (services) of a firm. The following problem of opinion control is studied. problems of the opinions control on networks are studied. A dynamic (difference) game in normal form where the players solve the problem of maximization of the sum of opinions of the members of a target audience by means of the closed-loop strategies of impact to the current opinions of the members of strong subgroups. We received the analytical solutions and conducted their comparative analysis.

The following directions of the further research are seemed to be interesting:

- analysis of the Stackelberg games on networks that reflect a hierarchy in marketing channels;

- analysis of the dynamic games in the form of characteristic function that model the cooperation of players and the ways of allocation of the received income;

- development of the methods of identification of the game theoretic models in specific applications.

\section{References}

Agieva, M. T., Korolev, A.V., Ougolnitsky, G.A. (2019). Modeling and Simulation of Impact and Control in Social Networks. Modelling and Simulation of SocialBehavioural Phenomena in Creative Societies: First International EURO Mini Conference, MSBC 2019. Vilnius, Lithuania, September 18-20, 2019 Proceedings. N. Agarwal, L. Sakalauskas, G.-W. Weber (Eds.). Communications in Computer and Information Science 1079. Springer, 2019. pp. 29-40.

Agieva, M. T., Korolev, A. V., Ougolnitsky, G. A. (2020). Modeling and Simulation of Impact and Control in Social Networks with Application to Marketing. Mathematics, 8(9), 1529 .

Aleskerov, F. T., Blagoveshchensky, N. Yu., Satarov, G.A., et al. (2007). Influence and structural stability in the Russian Parliament (1905-1917 and 1993-2005 years).: Moscow.- 312 p. (in Russian).

Chkhartishvili, A. G., Gubanov, D.A., Novikov, D. A. (2019). Social Networks: Models of information influence, control and confrontation. Studies in Systems, Decision and Control, 189, pp. 1-158.

Chwe, M. S. (2000). Communication and Coordination in Social Networks. Review of Economic Studies, 67, 1-16.

De Groot, M.H. (1974). Reaching a Consensus. Journal of American Statistical Association, 69, 118-121.

De Marzo, P., Vayanos, D., Zwiebel, J. (2003). Persuasion Bias, Social Influence and Unidimensional Opinions. Quarterly Journal of Economics, 118(3), 909-968.

French, J., R. (1956). A formal theory of social power. The Psychological Review, 63, 181-194.

Godes, D., Mayzlin, D. (2004). Using Online Conversations to Study Word of Mouth Communication. Marketing Science, 23, 545-560.

Goldenberg, J., Libai, B., Muller, E. (2001). Talk of the Network: A Complex Systems Look at the Underlying Process of Word-of-Mouth. Marketing Letters, 2, 11-34.

Golub, B., Jackson, M. (2010). Naive Learning in Social Networks and the Wisdom of Crowds. American Economic Journal: Microeconomics, 2(1), 112-149. 
Gubanov, D. A., Novikov, D. A., Chkhartishvili, A. G. (2011). Informational influence and informational control models in social networks. Automation and Remote Control, 72(7), 1557-1567.

Harary, F. (1959). A Criterion for Unanimity in French's Theory of Social Power. Studies in Social Power. Michigan: Institute of Sociological Research, pp. 168-182.

Hegselman, R., Krause, U. (2002). Opinion Dynamics and Bounded Confidence Models: Analysis and Simulation. Journal of Artificial Societies and Social Simulation, 5(3).

Jackson, M. (2008). Social and Economic Networks. Princeton University Press. - 504 p.

Jackson M., Wolinsky, A. (1996). A strategic model of social and economic networks. J. Economic Theory, 71(1), 44-74.

Jackson, M., Zenou, Y. (2014). Games on networks. Handbook of game theory. Eds. Young, P., Zamir, S. Amsterdam: Elsevier Science, pp. 95-163.

Jorgensen, S., Zaccour, G. (2004). Differential Games in Marketing. Kluwer Academic Publishers, -176 p.

Jorgensen, S., Zaccour, G. (2014). A Survey of Game-Theoretic Models of Cooperative Advertising. European J. of Operations Research, 237(1), 1-14.

Korolev, A. V., Ougolnitsky, G. A. (2020). Optimal resource allocation in the difference and differential Stackelberg games on marketing networks. J. of Dynamics and Games, $7(2), 141-162$.

Noviko, D. A. (2014). Games and networks. Automation and Remote Control, 75(6), 11451154.

Roberts, F. (1976). Discrete Mathematical Models, with Applications to Social, Bio-logical and Environmental Problems. Prentice-Hall: Englewood Cliffs, NJ. 